\title{
Prostatectomy as a treatment for canine prostate cancer: a literature review
}

\author{
Jelle Stans*
}

\author{
Institute for Globally Distributed Open Research and Education, Beringen, Belgium
}

\begin{abstract}
Canine prostate cancer is a relatively rare condition with a poor prognosis. Both total and partial prostatectomy have been described as treatment strategies for this condition. Based on the available literature, it is clear that prostatectomy is usually employed in combination with other therapeutic strategies. However, it is apparent that the procedure is currently not suitable for curative intent. Its role as a palliative therapy has been better established. Among others, urinary incontinence and urinary tract infections were reported as important complications. The reported frequencies varied per study. Future research is needed to establish the role of prostatectomy in the treatment of canine prostate cancer. Based on the current lack of prospective studies, it is difficult to state whether prostatectomy should become a routine practice or first-line standard of care. Novel protocols for treating canine prostate cancer should be established. Keywords: Cancer, Carcinoma, Dogs, Prostate, Prostatectomy.
\end{abstract}

\section{Introduction}

Canine prostate cancer is a relatively rare condition that accounts for less than $1 \%$ of all cancers diagnosed in dogs (Obradovich et al., 1987; Bryan et al., 2007). Out of 431 dogs with prostatic conditions diagnosed in a Dutch center, 56 suffered from prostatic carcinoma (Teske et al., 2002). Several forms have been identified, of which adenocarcinoma (AC), transitional cell carcinoma (TCC), and undifferentiated cell carcinoma are the most common (Bennett et al., 2018; Cunto et al., 2019).

Canine prostate cancer has a poor prognosis due to the aggressive nature of this kind of tumors (both locally and systematically) (Cornell et al., 2000; Cunto et al., 2019). Untreated animals usually live for around one month (Griffin et al., 2018). One study reported an $80 \%$ metastatic rate at necropsy, mainly in lymph nodes, lungs, and bones (Cornell et al., 2000). Another important factor is the lack of response to the treatment strategies used to manage the condition (Griffin et al., 2018).

Several treatment strategies are used in the management of canine prostate cancer. Medical therapy, radiation therapy, and surgery (total and partial prostatectomy) have all been used to treat animals suffering from the condition. However, no first-line standard of care has been defined (Griffin et al., 2018).

Prostatectomy is a surgical technique that is used as one of the treatment strategies for prostate cancer (Griffin et al., 2018). Total prostatectomy (complete removal of the prostate gland) is, however, not routinely performed, partly due to the risk of serious complications after performing the procedure and due to the uncertainty about its added value compared to partial prostatectomy (White, 2000; Leroy and
Northrup, 2009). Partial prostatectomy has been suggested as a palliative treatment to relieve symptoms (L'Eplattenier et al., 2006). However, in general, literature regarding prostatectomy for the treatment of prostate carcinoma is relatively scarce.

The main goal of the present article is to summarize the available literature regarding the use of prostatectomy as a treatment for canine prostate cancer. Special attention will be given to studies investigating the therapeutic effectiveness and complications following the intervention. Additionally, gaps in the current knowledge are identified and proposals for future research will be formulated.

\section{Surgical procedures}

Total and partial prostatectomies are the two main types of surgery that can be performed in dogs with prostate cancer.

Total prostatectomy refers to the complete removal of the prostate gland and prostatic urethra. This procedure has been described in case reports and other literature (Bennett et al., 2018; Przadka et al., 2019). Some common features are summarized. In the first step, a urinary catheter is inserted into the urethra, followed by a midline laparotomy to open the abdominal cavity. Afterwards, the exposed vasa deferentia are ligated and cut. The tissues surrounding the prostate are subsequently dissected, as close to the organ as possible. To allow for transection of the urethra, the urinary catheter is (partly) withdrawn. The pre-prostatic urethra is then cut. After further dissection, the post-prostatic urethra is also cut circumferentially, after which the prostate is removed through the incision. Several urinary anastomosis techniques have been described, including uretero-urethral and cystourethral variants (Bennett et al., 2018). The abdominal incision is closed in three layers in a standard fashion. 
In 2016, a case report was published (Bacon et al., 2016) which described a total prostatectomy combined with cystectomy (total cysto-prostatectomy) in two dogs. In this report, the uretero-urethral anastomosis technique was used.

The technique of partial prostatectomy is described in several articles (L'Eplattenier et al., 2006; Vlasin et al., 2006). The prostate is also approached using a midline laparotomy. The reported studies used a urinary catheter to localize the urethra during the procedure. In both instances, a subtotal intracapsular approach was used. A part of the prostatic parenchyma was removed, and the capsule was closed afterward. During a partial prostatectomy, the prostatic urethra is not necessarily removed, meaning that an anastomosis is not always required.

In a study conducted in 2004 (Liptak et al., 2004), transurethral resection (TUR) was evaluated in dogs, including three suffering from prostatic carcinoma. After opening of the abdominal cavity, a ventral cystotomy was performed to assess the prostatic urethra and bladder neck. Afterwards, TUR of the prostatic urethra was performed with a cauterized cystoscopic loop. In this study, radiotherapy was also administered intraoperatively. Afterwards, the bladder and abdominal incisions were closed in a standard fashion.

The current paper focused on the basic technical aspects of the different procedures. More detailed information about the techniques, the analgesia used, and other aspects can be found in the original articles.

\section{Therapeutic effectiveness}

The literature describing prostatectomy used for the treatment of canine prostate cancer was screened for information regarding the effectiveness in extending the survival time of animals. Key information of these studies is summarized in Table 1.

A number of case reports have been published describing single animals suffering from prostate cancer undergoing prostatectomy (Bacon et al., 2016; Przadka et al., 2019). These reports mostly focus on the technique and its execution, but they also contain information about the disease and clinical outcome. The case report of Bacon et al. (2016) describes the execution of total cysto-prostatectomy (total removal of both bladder and prostate) in two dogs, one of which suffered from prostatic carcinoma. After diagnosis, the dog underwent eight months of 3-weekly chemotherapy before undergoing total cysto-prostatectomy due to the progression of disease. No signs of metastatic disease were found. Around twelve days after surgery, the animal was euthanized due to symptoms, some of which were related to aspiration pneumonia and not tumor itself. No recurrence or metastasis of the prostate carcinoma was found during necropsy. In 2019, a case report was published about a total prostatectomy performed in a dog suffering from prostatic AC (Przadka et al., 2019). The animal underwent a total prostatectomy after at least 3 weeks of difficulty urinating. No evidence of metastasis was present. After the surgery, the dog was treated with analgesics, including meloxicam. The animal died within 6 weeks after the surgery due to unknown reasons.

In 2006, a case series was published that described partial prostatectomy using a neodymium-doped yttrium aluminum garnet (Nd:YAG) laser in 12 dogs, of which 8 suffered from prostatic carcinoma (L'Eplattenier et al., 2006). In three of these dogs, metastasis was either discovered during surgery or metastasis developed during follow-up. After the surgery, these animals were treated with meloxicam and IL-2. The median survival time of all the animals with prostatic carcinoma was 103 days. In five animals that clinically benefitted from the surgery, the median survival time was 183 days. The other three animals died within 16 days. A more recent retrospective case series investigated the outcomes of total prostatectomy in 25 dogs suffering from prostatic carcinoma. (Bennett et al., 2018). A total of $21(81 \%)$ of these dogs were also treated with other therapies, mainly nonsteroidal anti-inflammatory drugs (NSAIDs) and/or chemotherapy (mitoxantrone or carboplatin). Nineteen animals (76\%) died due to the cancer, while three animals were still alive at the end of the study. The three remaining dogs were euthanized for reasons that were not directly related to the cancer. The median disease-free interval for animals where data were available was 81.5 days and was longer for dogs with (at least suspected) local recurrence than for dogs with (at least suspected) metastatic disease (85 $v s$. 76, respectively). One year after surgery, 32\% of the animals were still alive, while only $12 \%$ were alive after two years.

Only two clinical trials assessing the effectiveness of prostatectomy were identified (Liptak et al., 2004; Vlasin et al., 2006). In the trial of Liptak et al. (2004), six dogs underwent TUR, of which three suffered from prostatic carcinoma. Two of these dogs were diagnosed with prostatic TCC and did not show metastases. The last dog suffered from undifferentiated prostate carcinoma with lymph node metastasis. In addition to the TUR, two of the dogs were treated with intraoperative radiotherapy (IORT). The survival times of the three dogs with prostatic carcinoma ranged from 32 to 264 days. The authors state that it is possible that the TUR did not provide an additional overall survival advantage over the rest of the treatments administered. In 2006, a prospective clinical trial comparing subtotal intracapsular prostatectomy and total prostatectomy as a palliative treatment for advanced-stage prostatic cancer was conducted (Vlasin et al., 2006). Ten dogs underwent subtotal prostatectomy while eleven dogs underwent total prostatectomy. Dogs undergoing a subtotal prostatectomy survived, on average, significantly longer than dogs undergoing total prostatectomy (112 vs. 19.2 days, respectively). Three additional dogs were euthanized on the request of the owners. 
From the aforementioned studies, it is clear that prostatectomy is usually employed with other therapeutic strategies. However, due to the administration of additional or adjuvant therapy next to the prostatectomy, it is difficult to assess the contribution of prostatectomy to overall survival. Indeed, in all the reported studies, most animals were treated with NSAIDs and/or chemotherapy. However, the average survival time of the four animals that did not receive additional treatment in the study of Bennett et al. (2018) was only 70.25 days. This seems lower than the overall median survival time of 231 days in the same study. However, only four out of 25 dogs were not treated with adjuvant therapy and the protocols were very variable. This makes it difficult to draw conclusions regarding the role of the different treatment strategies in prolonging the survival time.

Based on the survival outcomes in the studies reported, it seems highly unlikely that prostatectomy is a viable option for curative-intent treatment in most instances of canine prostate cancer. However, prostatectomy could be part of a curative therapy in non-metastasized prostate cancer. No studies specifically investigated this theory. Additionally, it is clear that prostatectomy can play an important role as a palliative treatment, both in metastasized and non-metastasized disease, to extend the survival time.

\section{Complications}

Total prostatectomy is a procedure that carries a high risk for complications and unwanted side effects. This is one of the main reasons why prostatectomy is not routinely performed (Leroy and Northrup, 2009). Information regarding the complications of total and partial prostatectomy mentioned in the reported studies is summarized in Table 2. Special attention is given to urinary incontinence, as it is the most frequently described complication.

Urinary incontinence has been described as a potentially serious complication of prostatectomy in dogs (Hardie et al., 1984; Basinger et al., 1987; Goldsmid and Bellenger, 1991). Estimates of urinary incontinence after total prostatectomy of $33 \%-100 \%$ have been mentioned (Goldsmid and Bellenger, 1991; Freitag et al., 2007; Bennett et al., 2018). Some studies do not mention occurrence of urinary incontinence (L'Eplattenier et al., 2006). In the study of Bennett et al. (2018), 8 out of $25(32 \%)$ that underwent total prostatectomy suffered permanent urinary incontinence. In the case report of Bacon et al. (2016), the dog undergoing total cystoprostatectomy suffered from urinary incontinence. This was, however, also present before the procedure. The animal that underwent total prostatectomy in the case report of Przadka et al. (2019) did not suffer from urinary incontinence. There was no report of urinary incontinence in dogs with prostate cancer undergoing partial or subtotal prostatectomy in several selected studies (Liptak et al., 2004; L'Eplattenier et al., 2006). It has been suggested that prostatic cancer itself has a role 


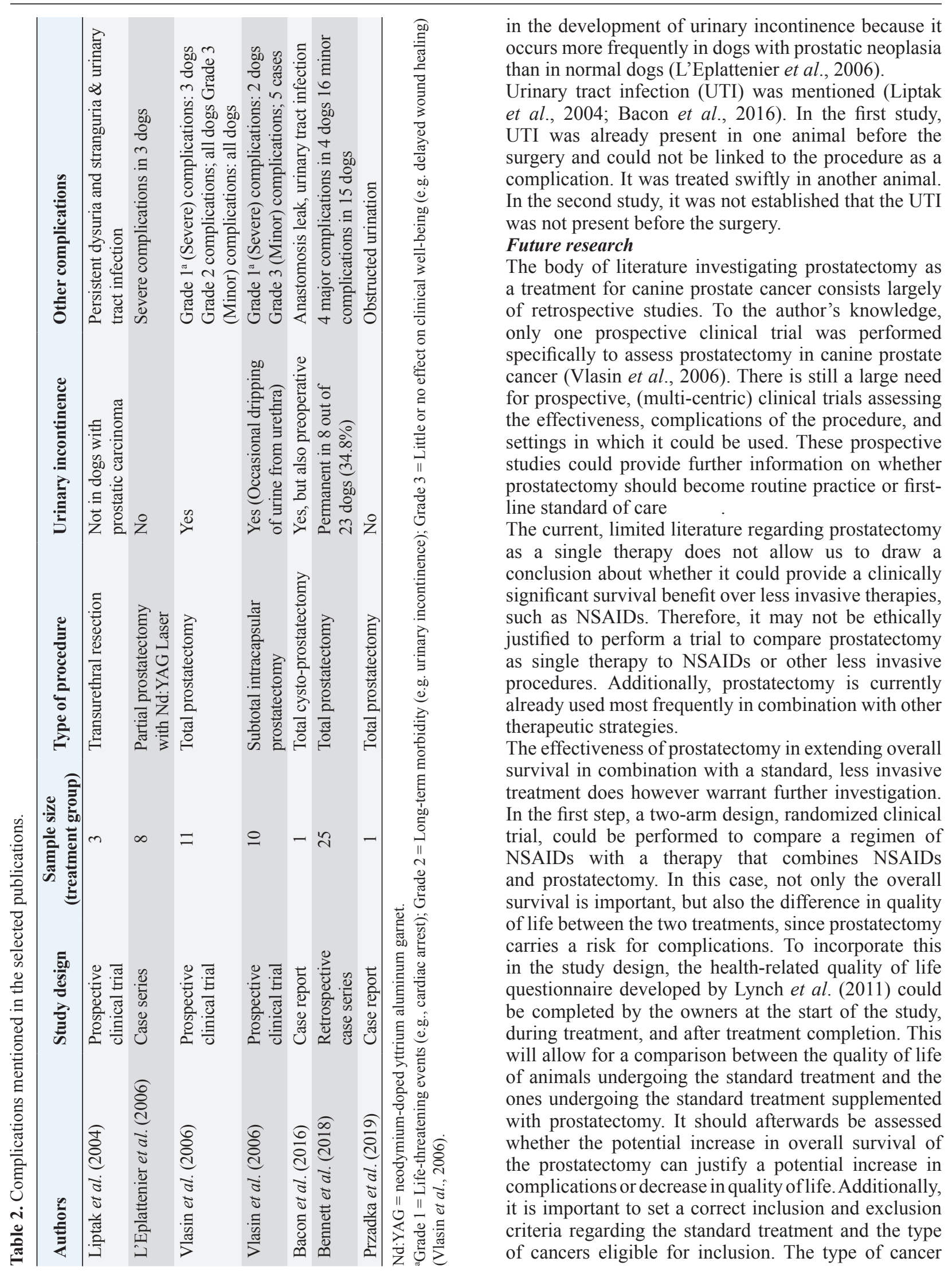


could be limited to a histological subgroup (e.g., AC or TCC) or to intra- or extracapsular disease. Finally, a decision needs to be made whether to test partial or total prostatectomy. Alternatively, two separate clinical trials or a three-arm design could be used to test both procedures.

It has been suggested that total prostatectomy could be used in non-metastasized prostate cancer (Leroy and Northrup, 2009), possibly with curative intent. To investigate this hypothesis, a single-arm study could be performed. Animals with confirmed prostatic neoplasia could be enrolled when there is no evidence of metastasis. This should be confirmed during the procedure itself. Animals in this study should be preventively treated with NSAIDs and/or chemotherapy to reduce the chance of recurrence as much as possible. The main outcome of this study would be whether or not there is recurrence. Secondary outcomes that could be considered are time to recurrence, overall survival, and quality of life before and after the procedure.

There are some hurdles to the conduct of the both above-mentioned trials. Due to the rarity of the disease, it may be difficult to achieve the required sample sizes within acceptable timelines. A similar effect can also be found in retrospective studies: in the study of Bennett et al. (2018), records from a time span of almost twelve years were searched to obtain 25 eligible animals. To circumvent this problem and to maximize external validity, the trials should be organized in multiple centers. Another potential issue is that owners may not consent to participation due to the invasiveness of the procedure. To address this concern, a nonrandomized design could be used or a cohort study could be designed. Both solutions will, however, lead to a reduction in internal validity.

There is currently little research into the factors that both veterinarians and owners consider to decide the optimal treatment strategy for canine prostate cancer. It is, therefore, not well understood whether owners and veterinarians feel that the potential benefits outweigh the risks of the potential complications of a prostatectomy. A better scientific understanding of these risks and benefits could allow veterinarians to better inform the owners, who can then make a better informed decision. Qualitative research could be carried out to investigate the considerations that are made by owners and veterinarians to decide whether or not to elect for a prostatectomy, and whether a total or partial removal of the organ should be performed.

\section{Conclusion}

The reported studies do not provide conclusive evidence that prostatectomy as a single therapy extends the survival time of dogs suffering from prostate cancer in a clinically significant way. Additionally, it is rarely used as a single therapy. It is, however, apparent that it does not constitute an option for curative treatment in almost all cases. Whether this is different in nonmetastasized disease or not remains to be elucidated. Based on the current literature, postoperative complications seem to be more frequent and severe following total prostatectomy than following partial prostatectomy. Urinary incontinence was a serious complication present in several dogs after total prostatectomy.

The body of literature investigating prostatectomy as a treatment for canine prostate cancer consists largely of retrospective studies. Prospective studies should be carried out to increase the understanding of this procedure. Based on the current evidence, it is difficult to state whether prostatectomy should become a routine practice or should be used a first-line standard of care. It is important that new protocols are developed to treat canine prostate cancer.

\section{Acknowledgments}

The author would like to thank E. Starczewska and F. Van Cauwenberghe for their valuable feedback prior to submission. This article is dedicated to Rio and Bailo.

\section{Conflict of interest}

The author declares there is no conflict of interest.

\section{References}

Bacon, N., Souza, C.H. and Franz, S. 2016. Total cystoprostatectomy: technique description and results in 2 dogs. Can. Vet. J. 57, 141-146.

Basinger, R.R., Rawlings, C.A., Barsanti, J.A., Oliver, J.E. and Crowell, W.A. 1987. Urodynamic alterations after prostatectomy in dogs without clinical prostatic disease. Vet. Surg. 16, 405-410.

Bennett, T.C., Matz, B.M., Henderson, R.A., Straw, R.C., Liptak, J.M., Selmic, L.E., Collivignarelli, F. and Buracco, P. 2018. Total prostatectomy as a treatment for prostatic carcinoma in 25 dogs. Vet. Surg. 47, 367-377.

Bryan, J.N., Keeler, M.R., Henry, C.J., Bryan, M.E., Hahn, A.W. and Caldwell, C.W. 2007. A population study of neutering status as a risk factor for canine prostate cancer. Prostate 67, 1174-1181.

Cornell, K., Bostwick, D., Cooley, D., Hall, G., Harvey, H., Hendrick, M., Pauli, B.U., Render, J.A., Stoica, G., Sweet, D.C. and Waters, D. 2000. Clinical and pathologic aspects of spontaneous canine prostate carcinoma: a retrospective analysis of 76 cases. Prostate 45, 173-183.

Cunto, M., Mariani, E., Guido, E., Ballotta, G. and Zambelli, D. 2019. Clinical approach to prostatic diseases in the dog. Reprod. Domes. Anim. 54, 815-822.

Freitag, T., Jerram, R., Walker, A. and Warman, C. 2007. Surgical management of common canine prostatic conditions. Compend. Contin. Edu. Vet. 29, 656-658. 
Goldsmid, S. and Bellenger, C. 1991. UrinaryIncontinence after prostatectomy in dogs. Vet. Surg. 20, 253-256.

Griffin, M.A., Culp, W.T.N. and Rebhun, R.B. 2018. Lower urinary tract neoplasia. Vet. Sci. 5(4), 96. doi:10.3390/vetsci5040096.

Hardie, E., Barstani, J. and Rawlings, C. 1984. Complications of prostatic surgery. J. Am. Anim. Hosp. Assoc. 20, 50-56.

L'Eplattenier, H., Van Nimwegen, S., Van Sluijs, F. and Kirpensteijn, J. 2006. Partial prostatectomy using Nd: YAG laser for management of canine prostate carcinoma. Vet. Surg. 35, 406-411.

LeRoy, B. and Northrup, N. 2009. Prostate cancer in dogs: comparative and clinical aspects. Vet. J. 180, 149-162.

Liptak, J., Brutscher, S., Monnet, E., Dernell, W., Twedt, D., Kazmierski, K., Walter, C.U., Mullins, M.N., Larue, S.M. and Withrow, S. 2004. Transurethral resection in the management of urethral and prostatic neoplasia in 6 dogs. Vet. Surg. 33, 505516.

Lynch, S., Savary-Bataille, K., Leeuw, B. and Argyle, D. 2011. Development of a questionnaire assessing health-related quality-of-life in dogs and cats with cancer. Vet. Comp. Oncol. 9, 172-182.

Obradovich, J., Walshaw, R. and Goullaud, E. 1987. The influence of castration on the development of prostatic carcinoma in the dog. 43 cases (19781985). J. Vet. Int. Med. 1, 183-187.

Przadka, P., Liszka, B., Piatek, A., Skrzypczak, P., Dzimira, S., Nizanski, W. and Kielbowicz, Z. 2019. Total prostatectomy combined with urethral anastomosis in a dog: a case report. Vet. Med. 64, 280-286.

Teske, E., Naan, E., van Dijk, E., Van Garderen, E. and Schalken, J. 2002. Canine prostate carcinoma: epidemiological evidence of an increased risk in castrated dogs. Mol. Cell. Endocrinol. 197, 251255.

Vlasin, M., Rauser, P., Fichtel, T. and Necas, A. 2006. Subtotal intracapsular prostatectomy as a useful treatment for advanced-stage prostatic malignancies. J. Small Anim. Pract. 47, 512-516.

White, R. 2000. Prostatic surgery in the dog. Clin. Tech. Small Anim. Pract. 15, 46-51. 